\title{
REVISTA BRASILEIRA DE ESTUDOS URBANOS E REGIONAIS
}

Publicação quadrimestral da Anpur

Volume 20, número 1, Janeiro-Abril de 2018

EDITOR RESPONSÁVEL

José Tavares Correia de Lira (FAU-USP)

EDITORA ASSISTENTE

Maria Cristina da Silva Leme (FAU-USP)

COMISSÃO EDITORIAL

Bernardo Campolina (CEDEPLAR-UFMG), Fania Fridman (IPPUR-UFRJ), Fernanda Sánchez (PPGAU-UFF), Helena Lucia Zagury Tourinho (PPDMUUNAMA), Maria Laura Silveira (CONICET/IG-UBA, Argentina), Maria Lucia Refinetti Martins (FAU-USP),

CONSELHO EDITORIAL

Alain Lipietz (Centre National de la Recherche Scientifique, França), Ana Fani Alessandri Carlos (PPGH-USP),

Andreas Novy (Vienna University of Economics and Business, Áustria), Anne-Marie Broudehoux (Université du Québec à Montréal, Canadá),

Benny Schvarsberg (PPG-FAU-UnB), Bernardo Mançano Fernandes (Geografia-UNESP/PP), Carlos Antônio Brandão (IPPUR-UFRJ),

Denise Elias (ProPGeo-UECE), Edesio Fernandes (DPU-Associates, England), Edna Castro (NAEA-UFPA), Ester Limonad (POSGEO-UFF),

Ivo Marcos Theis (PPGDR-FURB), José Aldemir de Oliveira (PPGSCA-UFAM), Jose Tavares Correia de Lira (FAU-USP), Mariana Fix (IE-UNICAMP),

Ramón Gutiérrez (Centro de Documentación de Arquitectura Latinoamericana, Argentina), Roberto Luiz do Carmo (NEPO-UNICAMP),

Sarah Feldman (IAU-USP), Sergio González López (Universidad Autónoma del Estado de México, México)

\section{COLABORADORAS/ES}

Alice Poma (UNAM), André Lemos (UFBA), Aristides Monteiro Neto (IPEA), Beatriz Helena Nogueira Diogenes (UFC),

Claudio Luis de Camargo Penteado (UFABC), Diana Lan (UNCPBA, Argentina), Felipe Sudré (UFMG), Germán Escobar (RIMISP),

Jorge Ramón Montenegro Gómez (UFPR), Josefina Di Nucci (UNCPBA, Argentina), Juliano Pamplona Ximenes Ponte (UFPA),

Luiz Antonio Fernandes Cardoso (UFBA), Luiz Eduardo Fontoura Teixeira (UFSC), Philippe Plas (Universite Paris 13), Regina de Souza Maniçoba (UnB), Renata Klautau Malcher de Araujo (Universidade do Algarve), Ricardo José Batista Nogueira (UFAM), Valdeci Monteiro dos Santos (UNICAMP), Carolina Maria Pozzi de Castro (UFSCar), Sidney Piochi Bernardini (UNICAMP), Leila Christina Dias (UFSC), Anete Brito Leal Ivo (UFBA), Martín Alberto Carné (UNL, Argentina), Marcelo Sili (UNS, Argentina), Carlos Lobo (UFMG), Paula Vilhena Carnevale Vianna (UNIVAP)

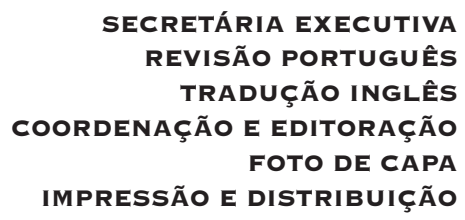

Wilma Guedes de Lucena (PPGG-UFPB)

Carlos Gomes de Castro e Roberta Menezes Couceiro

Brian Honeyball

Rian Narcizo Mariano

Foto de Paulo Couri

Letra Capital Editora (www.letracapital.com.br)

Indexada na Asociación de Revistas Latinoamericanas de Arquitectura (ARLA); na Bielefeld Academic Search Engine (BASE); no Centro de Referência e Informação em Habitação (InfoHAB); no Google Acadêmico; no Portal de Periódicos da ABEC (PoPA); no Portal de Periódicos da CAPES; no Public Knowledge Project (PKP Index); na Red de Revistas Científicas de América Latina y el Caribe, Espańa y Portugal (REDALYC); na Red Iberoamericana de Innovación y Conocimiento Científico (REDIB);

e no Sistema Regional de Información en Línea para Revistas Científicas de América Latina, el Caribe, Espańa y Portugal (Latidnex).

Dados Internacionais de Catalogação na Publicação (CIP) (Câmara Brasileira do Livro, SP, Brasil)

Revista Brasileira de Estudos Urbanos e Regionais - v.20 n.1,

2018. - Associação Nacional de Pós-Graduação e

Pesquisa em Planejamento Urbano e Regional; editor

responsável José Tavares Correia de Lira: A Associação, 2018

v.

Quadrimestral.

ISSN 1517-4115

O n 1 foi publicado em maio de 1999.

1. Estudos Urbanos e Regionais. I. ANPUR (Associação

Nacional de Pós-Graduação e Pesquisa em Planejamento

Urbano e Regional). II. José Tavares Correia de Lira.

711.4(05) CDU (2.Ed.) USP

711.405 CDD (21.Ed.) BC-2001-098 